\title{
Study of Surface Wave and Transmission Properties in Radome Surface
}

\author{
Varsha Mishra, Filippo Costa, Agostino Monorchio* \\ Dipartimento di Ingegneria dell'Informazione, \\ Università di Pisa - Pisa, Italy, \\ and RaSS National Laboratory, CNIT, Pisa, Italy \\ email: varsha.mishra@ing.unipi.it, \{filippo.costa, agostino.monorchio\}@unipi.it
}

\begin{abstract}
Surface wave and the transmission properties of a radome are discussed. The analysis of transmission properties is based on the transmission line theory. The surface wave analysis of FSS based radome structure has been carried out in the ANSYS HFSS software 19.0. The analyzed radome surface consists of a lossless dielectric material $(\varepsilon=2.65)$ coated with a Frequency Selective Surface (FSS).
\end{abstract}

\section{Introduction}

Radome are most popularly used structure to protect the antenna from the hostile condition (such as, heat, humidity, and cold etc.,) especially in the military applications $[1,2]$. The $100 \%$ transmission of electromagnetic (EM) wave through radome is an ideal requirement for practical applicatins. Radome transmission coefficient is usually studied by using plane wave approximation. However, in practical scenarios, radome surfaces are situated in the near field region of the antenna (i.e., $\mathrm{R}<2 \mathrm{D}^{2} / \lambda$, where, $\mathrm{D}$ is the maximum dimension of antenna, and $\lambda$ is the operating wavelength) In this case, the antenna may cause excitation of surface wave in the radome which could eventually increase the mutual coupling of other antennas sharing the same protecting cover. The situation is schematically depicted in Fig. 1.

In literature, plenty of radome surfaces have been designed for optimizing and shaping the transmission properties of EM wave. For this purpose, FSS and metasurfaces are the most popularly used techniques [3, 4]. Till now, surface wave propagation has been usually analyzed in the substrate of antennas [5,6]. Their propagation is usually inhibited by using Electromagnetic Bandgap Surfaces (EBG) $[7,8]$ or by using lossy material [9].

In this paper, we analyze the surface wave supported by a radome structure which is placed in the near-field region of the antenna. Initially, we discuss the unit cell of the transmitting spatial filter which is suitable for the radome. Next, in section III, we discuss the results numerical results in terms of transmission coefficient and surface wave propagation.

\section{Analyzed structure}

The Frequency Selective Surface (FSS) structure employed as a radome is shown in Fig. 2. The FSS is printed on a lossless substrate $\left(\varepsilon_{\mathrm{r}}=2.65\right)$ of thickness (t) $1.5 \mathrm{~mm}$. The periodicity $(\mathrm{P})$ of unit cell is $32 \mathrm{~mm}$, the width $(\mathrm{w})$ between two adjacent unit cells is $6.75 \mathrm{~mm}$, the width of ring $(\mathrm{s})$ is $1.5 \mathrm{~mm}$ and the length of outer ring $\left(\mathrm{L}_{1}\right)$ is $18.5 \mathrm{~mm}$.

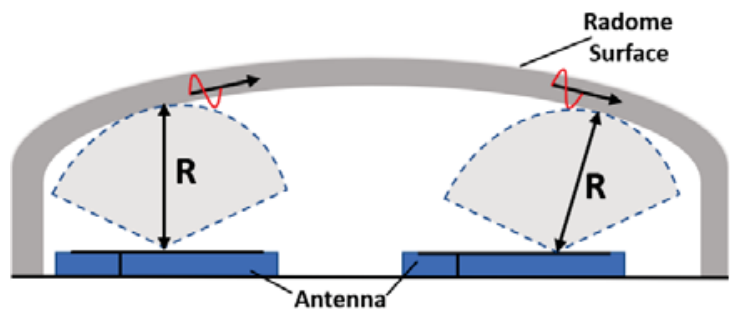

Figure 1. Radome is placed under the near-field region (R) of antennas.

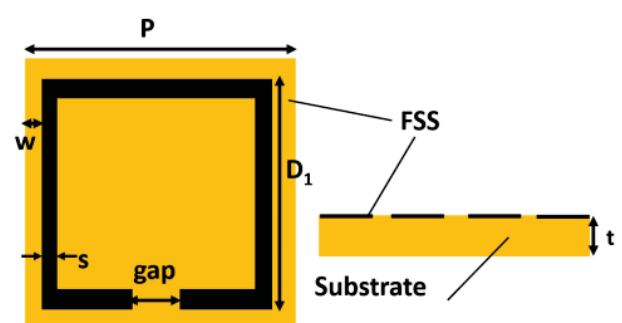

(a)

(b)

Figure 2. Analyzed unit cell (a) top view (b) side view.

\section{Numerical results}

The FSS has been simulated to calculate the transmission properties as a function of the gap which is varied from $1 \mathrm{~mm}$ to $5 \mathrm{~mm}$ as shown in Fig. 3. The designed FSS provides transmission properties in wide frequency range. The transmission band spans from 1.7 to $3.23 \mathrm{GHz}(1.53$ $\mathrm{GHz}$ ) for gap=1 mm, 2.1 to $4.45 \mathrm{GHz}(2.35 \mathrm{GHz})$ for gap=3 $\mathrm{mm}$, and 2.3 to $4.7(2.4 \mathrm{GHz})$ for gap $=5 \mathrm{~mm}$. Hence, as the gap varied from 1 to $5 \mathrm{~mm}$, bandwidth of the transmission band also increases from $1.53 \mathrm{GHz}$ to $2.4 \mathrm{GHz}$, respectively. The maximum bandwidth of $-1 \mathrm{~dB} \mathrm{~S}_{21}$ is 2.4 $\mathrm{GHz}$ (at gap $=5 \mathrm{~mm}$ ).

Other than the plane wave analysis, the proposed structure has been also analyzed in terms of surface wave propagation. The eigen mode solution for the selected unitcell FSS structure has been carried out in ANSYS HFSS software 19.0. Fig. 4 shows the k- $\beta$ dispersion diagram from 1.0 to $6.0 \mathrm{GHz}$ of frequency range which corresponds to the whole transmission band. As is evident, the FSS 
structure supports the propagation of surface waves in the transmission band of the radome. For this reason, the antenna coupling may be increased by the presence of the radome. The complete analysis of the antenna coupling will be shown at the conference.

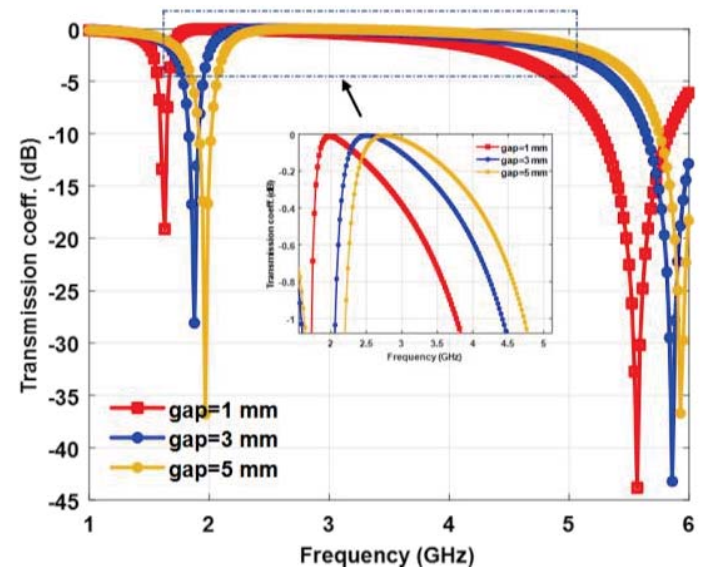

Figure 3. Transmission Coefficient (in $\mathrm{dB}$ ) of selected unit cell in TE polarized wave

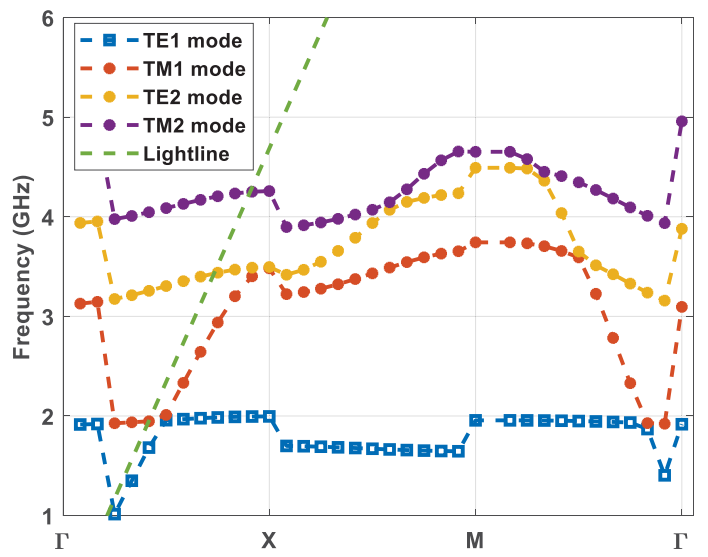

Figure 4. k- $\beta$ dispersion diagram in unit cell (in Fig. 1).

\section{Conclusion}

The surface wave properties of a pass-band radome has been analyzed. The existence of surface wave in the transmission band may cause the mutual coupling between antennas sharing the same radome cover.

\section{References}

1. K. Persson M. Gustafson "Reconstruction of equivalent currents using a near-field data transformation-With radome application" Progr. Electromagn. Res. (PIER) vol. 54 pp. 179-1982005.

2. Balanis, Constantine A. Advanced Engineering Electromagnetics. New York: Wiley, 1989.

3. F. Costa and A. Monorchio, "A Frequency Selective Radome With Wideband Absorbing Properties," in IEEE Transactions on Antennas and Propagation, vol. 60, no. 6, pp. 2740-2747, June 2012.

4. J. H. Kim, H. J. Chun, I. P. Hong, Y. J. Kim and Y. B. Park, "Analysis of FSS Radomes Based on Physical Optics
Method and Ray Tracing Technique," in IEEE Antennas and Wireless Propagation Letters, vol. 13, pp. 868-871, 2014

5. M. A. Khayat, J. T. Williams, D. R. Jackson, and S. A. Long, "Mutual coupling between reduced surface-wave microstrip antennas," IEEE Transactions on Antennas and Propagation, vol. 48, no. 10, pp. 1581-1593, Oct. 2000.

6. F. Costa, O. Luukkonen, C. R. Simovski, A. Monorchio, S. A. Tretyakov, and P. M. de Maagt, "TE surface wave resonances on high-impedance surface based antennas: analysis and modeling," IEEE Transactions on Antennas and Propagation, vol. 59, no. 10, pp. 3588-3596, 2011

7. D. Sievenpiper, Lijun Zhang, R. F. J. Broas, N. G. Alexopolous, and E. Yablonovitch, "High-impedance electromagnetic surfaces with a forbidden frequency band," IEEE Transactions on Microwave Theory and Techniques, vol. 47, no. 11, pp. 2059-2074, Nov. 1999, doi: $10.1109 / 22.798001$

8. O. Luukkonen, Má. G. Silveirinha, A. B. Yakovlev, C. R. Simovski, I. S. Nefedov, and S. A. Tretyakov, "Effects of Spatial Dispersion on Reflection From Mushroom-Type Artificial Impedance Surfaces," IEEE Transactions on Microwave Theory and Techniques, vol. 57, no. 11, pp. 2692-2699, Nov. 2009.

9. J. H. Richmond L. Peters Jr. R. A. Hill "Surface waves on a lossy planar ferrite slab" IEEE Trans. Antennas Propagat. vol. AP-35 pp. 802-808 July 1987. 